\title{
LA INICIATIVA PRIVADA EN UN SECTOR SINGULAR DEL ORDENAMIENTO SANITARIO
}

351.77

por

Enrique Argullol Murgadas

Doctor en Derecho

SUMARIO: I. INTRODUCCION.-II. EL ORDENAMIENTO SANITARIO Y LA REGULACION DE CEMENTERIOS.-III. LAS COMPETENCIAS MUNICIPALES EN ESTE CAMPO.-IV. LA INTERVENCION DE OTRAS ADMINISTRACIONES.-V. NOTAS SOBRE ALGUNOS ASPECTOS CONCRETOS.-VI. LA DIMENSION TERRITORIAL.

\section{INTRODUCCION}

Bajo el manto de la actual configuración del ordenamiento sanitario, se acoge una materia que presenta características peculiares y ha tenido una resonancia particular durante la historia espanola, especialmente al socaire de la llamada cuestión religiosa. El debate sobre confesionalismo y secularización se ha reflejado con especial intensidad - de forma apasionada muchas veces- en la ordenación de los cementerios. La tolerancia, o mejor, la libre convivencia, sólo en breves períodos ha presidido la gobernación del país. Más frecuente ha sido la presencia de orientaciones tendentes a dominar desde una visión parcial y proselitista los avatares de nuestra comunidad y la conciencia de sus miembros. $\mathrm{Y}$ el sectaris- 
mo en ocasiones ha querido proyectar su espectro más allá de la vida.

La cordura, que a intervalos ha inspirado los quehaceres colectivos, se ha concretado recientemente en la Ley 49/1978, de 3 de noviembre, sobre enterramientos en los cementerios municipales, que prohíbe expresamente toda discriminación religiosa o ideológica en este ámbito, al tiempo que confirma la obligación municipal de establecer cementerios abiertos a toda la población en los términos en los que no hubiese instalaciones de este tipo. Señalemos, además, que esta Ley derogó expresamente la Ley de la Jefatura del Estado de 10 de diciembre de 1938 sobre secularización de cementerios, dictada en plena guerra civil y con enfático espíritu de cruzada, que, entre otras medidas, ordenaba la separación, restableciendo las "antiguas tapias», entre el sector católico y civil.

No es propósito de este trabajo estudiar las diversas vertientes que inciden en la regulación de los cementerios, sino que el objeto se limita a examinar la presencia de la iniciativa privada en este campo concreto de la actividad administrativa, que, evidentemente, no puede tener únicamente una lectura sacralizada. La experiencia de otros países, singularmente de aquellos inspirados en las nuevas andaduras iniciadas a partir de la reforma luterana, ofrecen rasgos extraños a nuestra tradición. Aunque debe añadirse de inmediato que en determinados círculos muy selectos por razón del dinero, la familia o el estado religioso, también en nuestro contexto se admitió la existencia de lugares privados de enterramiento.

Lógicamente, al enfocar hoy estos temas debe hacerse desde la perspectiva y las coordenadas en que está asentado actualmente nuestro ordenamiento, alejado por completo de planteamientos tridentinos.

\section{EL ORDENAMIENTO SANITARIO Y LA REGULACION DE CEMENTERIOS}

La regulación de la actividad de cementerios, más allá de normas generales de contenido esencialmente ideológico, a las que ya hemos hecho referencia, está contenida generalmente en las Leyes y Reglamentos sanitarios. Las principales normas vigentes son la Ley de 
Bases de la Sanidad nacional del 25 de noviembre de 1944 y el Reglamento de Policía Sanitaria y Mortuoria del 20 de julio de 19.74.

Antiguamente, los cementerios fueron propiedad eclesiástica, si bien a partir del siglo XvIII se encomendaron a los Ayuntamientos funciones de orden sanitario. Con la Ley de 8 de enero de 1845 se previó la construcción generalizada de cementerios municipales, si bien subsistieron cementerios dependientes de las autoridades religiosas. Durante el siglo XIX se dictaron diversas disposiciones, restringiendo las inhumaciones en iglesias y cementerios que estuvieran en el interior de pueblos. Así, una Real Orden de 18 de julio de 1887 prohibió los entierros fuera de los cementerios comunes, si bien se establecieron excepciones para ciertas personas o respecto de panteones osarios. Una Real Orden de 17 de marzo de 1928 precisó dicha orientación al decir «que, manteniendo el principio de prohibición general para enterrar fuera de los cementerios públicos, pueda, sin embargo, el Gobierno autorizar inhumaciones en criptas y cementerios particulares, cualquiera que sea su emplazamiento, previo cumplimiento riguroso, en cada caso, de las disposiciones sanitarias vigentes, y de manera especialísima de aquellas que la Dirección General de Sanidad juzgue pertinentes en cada caso concreto".

Dejando al margen la polémica sobre cementerios católicos y civiles y la separación o no entre ellos, que es objeto de regulaciones contradictorias según sean las orientaciones políticas dominantes y que no nos ofrece un especial interés para examinar el tema que nos ocupa, se llega a la regulación legal aún vigente, que es la Ley de Bases de la Sanidad nacional, antes citada. La base 33, en lo que nos interesa, dispone que: "Todo Municipio tiene la obligación de disponer de uno o varios cementerios católicos de capacidad adecuada a su población para que la remoción de restos no sea necesaria antes de los diez años de enterramiento. Asimismo tendrá cementerios civiles independientes de los católicos. La autorización para la construcción de nuevos cementerios y ensanches o reformas de los antiguos corresponde a los Gobernadores civiles, respectivamente, previo informe de los Consejos provinciales de Sanidad del sitio en donde radiquen. La construcción de criptas o cementerios privados y concesión de enterramientos de este carácter en iglesias, edificios públicos o particulares será facultad de la Dirección Ge- 
neral de Sanidad. En los cementerios particulares, cualquiera que sea la entidad a que pertenezcan, la función de policía sanitaria corresponderá a la autoridad de este carácter del lugar en donde esté enclavado».

De las prescripciones de esta norma básica puede deducirse: a) el establecimiento de obligaciones a cargo de los Municipios, que más adelante examinaremos; b) la previsión de que junto a los cementerios municipales pueden existir otros cementerios que se califican como privados y particulares; se admiten asimismo criptas privadas; c) además de dichos cementerios privados o particulares, se prevén los entierros en iglesias y edificios públicos o privados, pudiéndose concluir que se trata de supuestos distintos; $d$ ) la autoridad que debe autorizar la construcción de los cementerios privados o particulares es superior a la competente en el caso de los cementerios municipales; $e$ ) previsión específica de funciones de policía sanitaria sobre cementerios particulares.

En desarrollo de la Ley de 25 de noviembre de 1944, y derogando la regulación reglamentaria anterior, se publicó el Reglamento de Policía Sanitaria Mortuoria de 22 de diciembre de 1960, que fue modificado por el Decreto 1713/1967, de 20 de julio. Por Decreto $2263 / 1974$, de 20 de julio, se publicó un nuevo Reglamento de Policía Sanitaria y Mortuoria, que es el texto vigente actualmente.

Los artículos 46 y siguientes regulan los diversos aspectos relativos a los cementerios. El artículo 47 recuerda la obligación municipal de disponer de un cementerio y admite la posibilidad de cementerios mancomunados, y el artículo 49 advierte de la necesidad de que los Planes de Urbanismo hagan las adecuadas precisiones. Los artículos 52 a 54 regulan las características técnicas de los cementerios -especialmente importante el primero, que se refiere a las condiciones de emplazamiento del cementerio y a las afectaciones de su entorno-, y los artículos 56 a 61 establecen el procedimiento para la construcción, ampliación y traslado y la administración de cementerios.

El Reglamento de 1974 se refiere a cementerios no municipales en diversas ocasiones. Así, el artículo 48 prevé concretamente que: «La Dirección General de Sanidad podrá autorizar la construcción de cementerios para comunidades exentas de la obligación de enterrar a sus miembros en los cementerios comunes si, al solicitarlo, se justifica debidamente tal condición. Dichos cementerios habrian 
de reunir los requisitos y obtener la autorización establecidos en los artículos 50 y siguientes».

Más adelante, al regular el procedimiento de construcción, ampliación y reforma de los cementerios, el artículo 55 establece que: «Los expedientes de construcción, ampliación y reforma de cementerios se instruirán por los Ayuntamientos con informe del Jefe local de Sanidad. Terminada la tramitación, expediente y proyecto se remitirán a la Jefatura Provincial de Sanidad, que, en unión de su informe, los elevará al Gobernador civil de la Provincia para su aprobación definitiva. La construcción, ampliación y reforma de cementerios particulares o privados, a que se refiere el párrafo tercero de la base 33 de la Ley de 25 de noviembre de 1944, habrá de cumplir los mismos requisitos y seguir la misma tramitación que las de los municipales, pero su aprobación se concederá por el Ministro de la Gobernación".

En los artículos 57 y 58, al regular la clausura de los cementerios, se dispone que: "Cuando las condiciones de salubridad y los planes de urbanización lo permitan, podrá el Ayuntamiento o entidad de quien el cementerio dependa iniciar expediente, a fin de destinar el terreno del cementerio, o parte de él, a otros usos. Para ello será indispensable el cumplimiento de las condiciones que resultan del texto de los artículos siguientes, además de lo dispuesto en el Reglamento de Bienes de las Entidades locales, si se trata de cementerio municipal. Con la finalidad indicada y también por razones sanitarias o de agotamiento transitorio o definitivo de su capacidad, previa resolución o autorización de la Jefatura Provincial de Sanidad, y proveyendo lo necesario al cumplimiento de lo dispuesto en el artículo 47, podrán suspender los enterramientos en cementerios concretos los Ayuntamientos y las entidades o particulares de que dependan». Asimismo, se prevé que: "Sin perjuicio de lo establecido por el Derecho canónico, corresponderá al Gobierno civil de la Provincia la competencia para autorizar la clausura de un cementerio municipal y el traslado total o parcial de los restos mortales que se hallen en él, previo informe de la Jefatura Provincial de Sanidad. En el supuesto de cementerios privados o particulares, dicha competencia corresponderá al Ministro de la Gobernación, previo informe de la Dirección General de Sanidad».

Finalmente, al regular algunos aspectos de la administración 
de cementerios, vuelve a recordarse la distinción entre cementerios municipales y privados. Concretamente, se establece que: «Tanto los cementerios municipales o mancomunados públicos en poblaciones de más de 10.000 habitantes, como los cementerios regulados en el párrafo tercero de la base 33 de la Ley de Sanidad nacional de 25 de noviembre de 1944, se regirán por su Reglamento de Régimen Interior, que será aprobado por el Gobernador civil de la Provincia, previo informe de la Jefatura Provincial de Sanidad. Los cementerios de poblaciones de más de 10.000 habitantes y los privados tendrán un encargado de su administración, designado por la autoridad municipal correspondiente o por la entidad o particular de quien dependan. El registro de cadáveres que se inhumen, exhumen $o$ incineren en el cementerio, en virtud de las licencias legales correspondientes, será llevado por la administración del mismo mediante libros donde consten los datos que se determinen por la Dirección General de Sanidad, mediante resolución publicada en el Boletín Oficial del Estado. La administración del cementerio comunicará al Jefe de Sanidad local, y en las capitales de Provincias, a la Jefatura Provincial de Sanidad, los datos reseñados en el libro registro, en la misma fecha en que se practiquen las anotaciones".

El examen de estos preceptos nos ofrece una regulación más bien compleja y con aspectos no completamente claros. Por una parte, el primero de los preceptos transcritos parece orientarse en el sentido de que existan dos tipos de cementerios, en función de la existencia o no de la obligación de enterrar en cementerios comunes: en consecuencia, se contempla la autorización de cementerios para "comunidades exentas de la obligación de enterrar sus miembros en cementerios comunes». Debe señalarse que se trata de círculos cerrados, ya que la posibilidad de entierro va ligada a la condición de miembro de la citada comunidad, expresión a la que no acompaña aclaración alguna y que no se repite en ningún otro precepto, a excepción del artículo 25 , al que aludiremos más adelante. La redacción del artículo 48 no permite, en principio, considerar como miembros de una comunidad exenta a los compradores de sepulturas construidas por una empresa mercantil. Conviene también advertir de que los demás preceptos, o bien se limitan a referirse a cementerios particulares o privados o que dependen de entidades o particulares, o bien hacen remisión a la ya citada base 33 . 
El artículo 61 contrapone los cementerios municipales o mancomunados, que califica como públicos, de forma muy redundante, puesto que no es concebible un servicio municipal privado a los particulares, pero sometidos ambos a la necesidad de que posean Reglamento de Régimen Interior, exigencia que podría dar lugar a interpretar que se contempla la existencia de una organización y una pluralidad de afectos, y que en el supuesto de cementerios particulares no se trata simplemente de una cuestión de ámbito familiar.

Debe tenerse en cuenta, por otra parte, que solamente en el caso de cementerios municipales se imponen, en caso de clausura, ciertas normas de publicidad para permitir a los familiares el traslado de los restos. Lógicamente, en el caso de los cementerios particulares no se prevé tal trámite, ya que la comunicación directa o interna se considera suficiente y no se trata, como en el caso de los municipales, de un servicio público. Debe también señalarse que todos los cementerios (art. 55) están sometidos a los mismos requisitos y procedimiento. Por ello, los cementerios particulares o privados no pueden confundirse con los panteones y entierros en edificios de otros usos, ya que en ningún caso éstos podrían cumplir los requisitos o condiciones -valga el ejemplo del emplazamiento alejado de núcleos urbanos- que todos los cementerios deben cumplir. La necesidad de que los cementerios particulares se adecuen a los requisitos establecidos para los cementerios municipales permite aclarar la referencia de la base 33 a la "construcción de criptas o cementerios privados», en el sentido de dos supuestos no equivalentes de instalaciones de titularidad privada, ya que los requisitos exigidos no son aplicables a las criptas. Asimismo, los requisitos técnicos establecidos en los artículos 52 y siguientes - proyecto de construcción, servicios, dependencias- hacen difícil su asimilación -nunca expresamente prevista- entre cementerio privado y cementerio familiar. Más bien parece que el carácter familiar se circunscribe a enterramientos de otro tipo.

Por otra parte, los artículos 25 y 27 del mismo Reglamento, al regular el carácter de las inhumaciones aportan también algunas precisiones. El primer precepto señala requisitos especiales para las inhumaciones que se hagan fuera de los cementerios comunes (no se habla de públicos o de municipales) o de comunidades exentas. El segundo precepto, al definir la condición de sepelios, lo hace 
según sean dentro del término municipal o del ámbito mancomunado, y no lo regula respecto al carácter municipal o público del cementerio. Debe también llamarse la atención sobre la redundancia que se produciría si se estableciera una estricta asimilación entre cementerios de comunidades exentas y cementerios privados o particulares: en efecto, el artículo 48 , por lo que se refiere a los primeros, regula los requisitos y el procedimiento, y el artículo 55, refiriéndose expresamente a cementerios particulares o privados, regula también las condiciones y procedimiento.

Como resumen del estudio de la reglamentación vigente en la actualidad pueden hacerse algunas consideraciones. En primer lugar, debe insistirse en que en nuestro ordenamiento la titularidad municipal de los cementerios es la regla general, si bien se admiten diversos supuestos especiales, en virtud de los cuales pueden autorizarse cementerios no municipales. En segundo lugar, parece que puede afirmarse que existen, desde el punto de vista de la titularidad, dos categorías de cementerios: públicos y privados o particulares. No obstante, desde la perspectiva de los usuarios, los cementerios pueden ser comunes o reservados a ciertos grupos o comunidades, cuyos componentes están eximidos de la inhumación en los primeros. Por otra parte, y teniendo en cuenta las formalidades del entierro, podría distinguirse entre los lugares donde se realizan las inhumaciones de carácter ordinario - los cementerios, bien sean comunes o de comunidades exentas - y otros lugares de entierro, como son iglesias, panteones, edificios. Partiendo de la anterior clasificación, parece que podrían señalarse los siguientes tipos de cementerios: municipales, que siempre son comunes; particulares o privados, que pueden ser comunes o de comunidades exentas; $y$, además, fuera de la categoría de los cementerios, los panteones y entierros en iglesias, edificios... En tercer lugar, es conveniente recordar que todos los cementerios deben cumplir los requisitos y condiciones mínimos fijados en la normativa. Asimismo, su construcción y explotación deberá regirse por las previsiones reglamentarias que en cada caso sean aplicables. Finalmente, debemos insistir en la ambiguiedad que presentan algunos preceptos reglamentarios, que podría hacer aconsejable que la Administración competente realizara las pertinentes aclaraciones normativas, en las que, a la vez, debería matizarse la regulación de los requisitos, condi- 
ciones o características técnicas de los cementerios que responden directamente a una problemática propia de los cementerios municipales.

\section{LAS COMPETENCIAS MUNICIPALES EN ESTE CAMPO}

El artículo 3 de la Ley 49/1978 ha reafirmado la obligación de los Municipios en orden a disponer de cementerios en sus términos. Es una confirmación reciente de un planteamiento constante de nuestra legislación local. En la letra c) del segundo párrafo del artículo 101 de la Ley de Régimen local, texto de 24 de junio de 1955, se prevé, entre las funciones municipales, los «cementerios y servicios fúnebres». El artículo 102, al señalar como obligación de todo Municipio la prestación del servicio de cementerio (letra e) ), configura una auténtica competencia municipal. En relación con este tema, interesa estudiar dos aspectos: alcance de la competencia municipal y formas de ejercicio de la competencia municipal.

Por lo que se refiere al primer aspecto, debe plantearse si la competencia municipal sobre cementerios implica la exclusión de la actividad privada en este campo, al margen de la que pueda producirse por encargo discrecional del Municipio. En principio, la inclusión de la materia de cementerios entre las obligaciones mínimas de los Municipios podría configurar una competencia exclusiva municipal. Ahora bien, las previsiones legales - concretamente, la Ley de Bases de la Sanidad nacional - sobre cementerios particulares, privados y de comunidades exentas permiten matizar el sen. tido de dicha exclusividad, que no puede impedir la presencia de la iniciativa particular. Por otra parte, la referencia del artículo 172 de la Ley de Régimen local, y en relación con el artículo 69 del Reglamento de Servicios de las Corporaciones locales de 17 de junio de 1955, a los cementerios, como servicios o actividades municipalizables, es una confirmación de que la atribución de competencia a los Municipios no excluye la actividad privada en este campo. Ahora bien, es necesario advertir que la consideración a hacer sería absolutamente distinta si el servicio de cementerios (que es diferente y no coincide con el de pompas fúnebres) estuviera municipalizado con monopolio (arts. 164 y ss. de la Ley de Régimen local y 45 y ss. del Reglamento de Servicios) en el Municipio donde 
se pretendiese construir el cementerio privado. En ese caso, la municipalización con monopolio excluiría totalmente la iniciativa privada.

En este orden de consideraciones, no debe olvidarse que en realidad existen cementerios no municipales de carácter común en muchos pueblos: son los conocidos como cementerios parroquiales. A ellos hacía referencia precisamente una antigua sentencia del Tribunal Supremo de 12 de abril de 1929, en la que se indicaba que si bien los Ayuntamientos estaban obligados a construir cementerios municipales, según disponía el artículo 203 del Estatuto municipal vigente entonces, no tenían la exclusiva de construirlos y explotarlos.

Parece, pues, que a la luz de la legislación local no existen obstáculos para la creación de cementerios por parte de la iniciativa privada, que deberán regirse por el ordenamiento general sanitario, a excepción del supuesto de aquellos Municipios que hubieran municipalizado el servicio de cementerios.

Como se ha dicho antes, existe un segundo aspecto en relación a la competencia municipal, por lo que se refiere a los cementerios, que interesa en el momento de examinar el papel de la iniciativa privada. La legislación local prevé que los servicios municipales pueden prestarse de manera directa por las propias Corporaciones locales o bien a través de la actividad de los particulares, que son quienes asumen la responsabilidad directa de la prestación. Pueden existir fórmulas mixtas a través del establecimiento de empresas con participación municipal y particular. El servicio de cementerio, de acuerdo con los artículos 156 y siguientes de la Ley de Régimen local y 67 y siguientes del Reglamento de Servicios, puede prestarse por gestión indirecta, es decir, por la iniciativa privada, a resultas de un encargo de la Corporación local. La fórmula más importante de gestión indirecta es la concesión, a través de la cual la iniciativa privada puede asumir la construcción de la obra y la explotación del servicio o únicamente dicho último aspecto (artículo 114 del Reglamento de Servicios). Cabe también, aunque tiene un alcance mucho más reducido, la fórmula del concierto, en virtud del cual el Ayuntamiento prestaría el servicio a que está obligado, a través de instalaciones propiedad de la iniciativa privada. La regulación de ambas fórmulas de gestión indirecta se encuentra esencialmente en los Reglamentos de Servicios y de Con- 
tratación de las Corporaciones locales. Sin entrar en un estudio detallado de las características de cada forma de gestión, es necesario, no obstante, destacar que, en el primer caso, los bienes e instalaciones afectas o que constituyen el cementerio permanecen de titularidad pública y el concesionario únicamente podria transmitir subconcesiones, y el cementerio debería construirse y explotarse según las condiciones propias de los cementerios municipales públicos. En cambio, en el segundo caso, no existe alteración en el carácter privado de la propiedad y es posible, por tanto, su transmisión. Esta ventaja, desde el punto de vista de la iniciativa priva$\mathrm{da}$, no puede sobrevalorarse, ya que esta fórmula tiene un alcance temporal concreto y, por otra parte, implica siempre la preexistencia del cementerio privado, con lo que se reconduce el tema a la principal cuestión estudiada.

En definitiva, desde el punto de vista de las competencias municipales, no hay obstáculos generales para la admisión de la iniciativa privada en la construcción y explotación de cementerios, pero deberá estarse a las determinaciones concretas que pueda haber adoptado cada Municipio en cuanto a la municipalización o no, y en régimen o no de monopolio, del servicio de cementerios.

Debemos advertir, finalmente, que el papel de los Municipios no se agota en la vertiente examinada hasta ahora, antes bien, se extiende también al hecho territorial que implica toda implantación en el espacio, en razón de las competencias urbanísticas municipales. A este aspecto nos referimos en apartado.

\section{LA INTERVENCION DE OTRAS ADMINISTRACIONES}

En la ordenación de los cementerios, y en razón de su trascendencia en el ámbito sanitario, se ha producido una progresiva intervención de la Administración estatal, que no sólo se ha concretado en la regulación común y superior de estas instalaciones, sino que ha supuesto asimismo su apoderamiento por lo que respecta a específicas funciones de policía. Siguiendo un esquema muy conocido, se ha producido un progresivo condicionamiento de la actividad municipal a través de ordenamientos sectoriales.

En nuestro sistema constitucional, la implantación de las Comunidades Autónomas ha producido una descentralización de fa- 
cultades legislativas, reglamentarias y administrativas, que se proyecta también en el orden sanitario, como ponen de relieve tanto el artículo 149.1 de la Constitución, que reserva necesariamente al Estado sólo las bases y coordinación de la sanidad interior, como los correlativos preceptos de los distintos Estatutos de Autonomía.

De forma específica, además, durante el período preautonómico, se efectuaron transferencias de funciones concretas en el ámbito sanitario, que tienen un particular interés en estas páginas, en cuanto se hizo en las mismas expresa referencia a la actividad que estamos estudiando.

En el examen de la intervención de Administraciones públicas no locales en la regulación de los cementerios, tomamos como punto de referencia la Comunidad Autónoma catalana. En todo caso, las conclusiones que se obtengan son válidas para otras Administraciones territoriales, y de tratarse de Comunidades que no ostentasen estas competencias, para la Administración estatal.

Como resultado del proceso abierto con el Real Decreto-ley de 30 de septiembre de 1977, la Generalidad de Cataluña es la Administración pública competente en materia de policía sanitaria mortuoria en el territorio de Cataluña. Para aclarar el alcance de las funciones de dicha institución es necesario hacer algunas consideraciones.

En primer lugar, debe señalarse que el Real Decreto 2210/1979, de 7 de septiembre, atribuye a la Generalidad la realización de todas las funciones administrativas (autorizaciones, inspección...) previstas en el Reglamento de Policía Sanitaria Mortuoria. Concretamente, el artículo 16, letra $c$ ), prevé que: «Se transfieren a la Generalidad...: c) Las competencias que, en relación con la policía sanitaria mortuoria, atribuyen el Decreto 2263/1974, de 20 de julio, y disposiciones complementarias, a los órganos de la Administración del Estado. Para asegurar la necesaria coordinación con las demás Entidades y órganos competentes en los supuestos de traslados de cadáveres cuyo recorrido exceda del territorio de la Generalidad, ésta deberá cumplir, en sus propios términos, las exigencias de comunicación previstas en el artículo 29 y en el apartado d) del artículo 36 de la citada disposición".

Estas funciones traspasadas tienen su más amplio alcance en el nivel administrativo o ejecutivo, ya que, como dice el artículo 15 
del mismo Decreto, las competencias traspasadas comprenden la organización, programación, dirección, resolución, control, vigilancia, tutela, sanción e intervención, así como la recopilación de datos e información.

Posteriormente, el Estatuto de Autonomía ha otorgado a la Generalidad facultades normativas. Concretamente, el artículo 17.3 dispone que: "Corresponde a la Generalidad de Cataluña el desarrollo legislativo y la ejecución de la legislación básica del Estado en materia de sanidad interior". Por consiguiente, dado que la sanidad mortuoria y la instalación de cementerios están comprendidas en el concepto de sanidad interior, corresponde también a la Generalidad dictar las Leyes y Reglamentos pertinentes, respetando, no obstante, las bases de la legislación estatal.

Por bases de la legislación estatal deben entenderse, según ha establecido, entre otras, la sentencia del Tribunal Constitucional del 18 de julio de 1982, los "principios o criterios básicos, estén o no formulados como tales», "los que racionalmente se deducen de la legislación reciente», sin que deba esperarse a que el Estado fije expresamente las «bases» para que la Generalidad pueda hacer uso de sus facultades normativas. En todo caso, en la actualidad podemos considerar que el núcleo de las «bases» se encuentra en la Ley de 1944.

En definitiva, a excepción del establecimiento de las «bases», en el sentido indicado, la Generalidad es la institución competente para regular legal y reglamentariamente y ejercitar las funciones administrativas en materia de sanidad mortuoria.

\section{NOTAS SOBRE ALGUNOS ASPECTOS CONCRETOS}

El primer aspecto a considerar en este apartado es el del área de influencia del cementerio. La vigente regulación de entierros, concretamente el artículo 27 del Reglamento de Policía Sanitaria Mortuoria, califica como sepelios ordinarios los que se efectúen dentro del término municipal o de los términos de los Municipios que se hayan mancomunado para construir un cementerio. En otro caso, se imponen ciertas autorizaciones, los féretros deben reunir condiciones especiales, y en algunos supuestos, los cadáveres deben ser objeto de tratamientos peculiares. En este sentido, al autorizarse 
un cementerio particular será necesario establecer su área de influencia, a los efectos que acabamos de señalar. En relación con este punto puede tener significado la Orden de 16 de junio de 1947, que para facilitar los entierros en Madrid y pueblos de su entorno consideraba dichos términos como un único casco.

En segundo lugar, debe insistirse en que los proyectos de construcción de cementerios deberán acomodarse, en principio, a las características técnicas reguladas en los artículos 51, 52, 53 y 54 del Reglamento de Policía Sanitaria y Mortuoria. Asimismo, como ya se ha dicho, deberían cumplirse las condiciones sobre emplazamiento y distancias.

\section{LA DIMENSION TERRITORIAL}

Naturalmente, la implantación de un cementerio incide en el territorio, por lo que habrá que examinar también su viabilidad desde la perspectiva de la Ley del Suelo y del Planeamiento Urbanístico.

Los Planes Generales de Ordenación deben prever con carácter general espacios para equipamientos de la población. El artículo 12.1.b) de la Ley del Suelo, texto de 9 de abril de 1976, así lo establece expresamente. El Reglamento de Planeamiento de 23 de junio de 1978 ha precisado, concretamente en su artículo 25, que el sistema general de equipamiento comunitario, que forma parte de la estructura general y orgánica del territorio, comprende los cementerios. Con ello, por otra parte, no hace sino recoger lo que prevé la legislación sanitaria y local.

Por tanto, la localización de los cementerios queda en manos de los Planes Generales o de acuerdo con el procedimiento de desarro. llo que los mismos se planteen. Así, si el Plan General establece sólo una reserva genérica de espacios para equipamientos comunitarios, a través de un Plan Especial puede fijarse el uso y establecer el de cementerio. Si se promueve únicamente por la iniciativa privada, debe pedirse, a tales efectos urbanísticos, la correspondiente licencia. Acabadas las obras, deberían solicitarse las licencias de ocupación y de apertura.

En otros supuestos en los que el Plan General o las Normas Subsidiarias señalan el uso de cementerio, para su implantación, si no 
se reserva como cementerio municipal, la iniciativa privada únicamente precisa de las licencias antes citadas.

Ahora bien, al lado de dicho procedimiento, la Ley del Suelo, al regular el suelo no urbanizable, prevé un régimen al que podrían acogerse, en las condiciones que citaremos, los cementerios, sin que las previsiones expresas del planeamiento sobre equipamientos, a que hemos aludido, impidan por sí mismas esa otra vía, aunque, lógicamente, deberá estarse a las determinaciones del planeamiento.

El artículo 85 de la Ley del Suelo establece las prescripciones esenciales del suelo no urbanizable, que no es objeto de especial protección, y de forma expresa por los Planes Generales. El artículo 44 del Reglamento de Gestión Urbanística de 25 de agosto de 1978 las ha desarrollado. En suelo no urbanizable, siempre que el uso no haya sido declarado incompatible por un Plan General o por unas Normas Subsidiarias de Planeamiento, de existir dichos documentos urbanísticos, pueden autorizarse, a través de un procedimiento especial, «instalaciones de utilidad pública o de interés social que hayan de emplazarse en el medio rural", siempre que las construcciones no sigan las pautas de los núcleos urbanos y se respete, por lo que se refiere a la dimensión de la propiedad, la legislación agraria. La tramitación de la autorización de un cementerio en suelo no urbanizable es compleja, de acuerdo con lo previsto en los artículos 85 y 44, antes citados. Inicialmente, debe formularse la petición ante el Ayuntamiento en cuyo territorio debe localizarse la edificación e instalación de utilidad pública. El escrito de solicitud debe ir acompañado de plano en el que se refleje la finca en cuestión, con expresión de sus medidas. También debe describirse la superficie ocupada por las edificaciones e instalaciones y características esenciales de las mismas. Por otra parte, deben justificarse tres extremos: la utilidad pública o interés social de las instalaciones, necesidad de emplazamiento en medio rural y no formación de núcleo de población. Este último punto, en el caso de un cementerio, obviamente, no tiene significación alguna.

Por lo que se refiere a la declaración de utilidad pública o interés social, debe advertirse que puede tener un triple origen: a) derivar de una norma preexistente referida a la actividad o bien a la institución que quiere llevarla a cabo; b) resolución expresa del propio Municipio, que deberá adoptarse en un expediente separado, 
y c) resolución de la Administración competente en el orden sanitario-mortuorio. Por lo que se refiere a la demostración de la necesidad de emplazamiento en medio rural, parece que la misma exigencia del Reglamento de Policía Sanitaria Mortuoria de que los cementerios queden situados, al menos, a 500 metros de núcleo habitado, puede ser una indicación a este respecto. 